\title{
Oesophageal symptoms, their causes, treatment, and prognosis in patients with the acquired immunodeficiency syndrome
}

\author{
G M CONNOLLY, D HAWKINS, J N HARCOURT-WEBSTER, P A PARSONS, \\ O A N HUSAIN, AND B G GAZZARD
}

From St Stephen's Hospital, London

SUMMARY In a prospective study of 154 AIDS patients, $48(31 \%)$ complained of pain on swallowing both liquids and solids and $32(21 \%)$ of these also had dysphagia. While candidiasis was the most common cause of symptoms ( 26 patients), discrete ulceration of the oesophagus occurred in 12 instances in 10 patients (four cytomegalovirus, four herpes simplex virus, three aphthous ulcer, one peptic ulcer). One patient had a diffuse oesophagitis caused by Mycobacterium avium intracellulare. No cause was found for the oesophageal symptoms in four patients. Kaposi's sarcoma (KS) was found in seven patients associated with other pathology in four. All 26 patients with oesophageal candidiasis only, also had oral involvement. All the patients with herpes simplex virus (four) and aphthous ulcers (three) had obvious perioral involvement. Three of the four patients with cytomegalovirus ulceration had evidence of disease elsewhere (colon or retina). All patients with Kaposi's sarcoma lesions had skin and buccal cavity involvement. The cause of oesophageal disease was usually obvious at endoscopy. The appearance of candidiasis was typical and the various ulcerating lesions also had different macroscopic configurations. Cytomegalovirus infection produced deep linear ulcers in the distal oesophagus, herpes simplex oesophagitis is similar in appearance to the typical perioral lesions of fluid filled vesicles. Diagnostic radiology was not helpful in most patients. In nine of 17 patients with candidiasis, the barium swallow examination performed within 24 hours of presentation was normal. In only three of seven patients with oesophageal ulceration (three cytomegalovirus, two herpes simplex virus, one aphthous, one peptic) was there evidence of an abnormality. Treatment produces symptomatic relief. All patients with candidiasis responded to ketoconazole, the four with herpes simplex virus to acyclovir and one of three with aphthous ulceration had a dramatic response to thalidomide. The three patients with cytomegalovirus infection who were treated with foscarnet had a prolonged remission of symptoms. The overall prognosis of patients with oesophageal symptoms is poor, with an average survival time from a definitive diagnosis of five months (range one to 13).

Since the first description of the acquired immune deficiency syndrome (AIDS) in 1981,' a major epidemic has occurred in the United Kingdom particularly among male homosexuals. ${ }^{2}$ Many AIDS patients present with gastrointestinal disorders including weight loss, diarrhoea, and oesophageal symptoms. ${ }^{3-5}$

Address for correspondence: Dr B G Gazzard, St Stephen’s Hospital, Fulham Road, London SW10 9TH.

Accepted for publication 8 December 1988.
The common causes of dysphagia in human immunodeficiency virus (HIV) infected patients are different from those usually encountered in gastroenterological practice and consist of a variety of opportunistic infections including candida, cytomegalovirus or herpes simplex virus. Kaposi's sarcoma may also affect the gullet. any of these abnormalities is sufficient for the diagnosis of AIDS to be confirmed in a patient who is HIV antibody positive. ${ }^{9}$ 
Although the present distribution of AIDS cases across the United Kingdom is patchy, the epidemic will spread and the advice of most gastroenterologists will be sought about these patients in the future. We have looked prospectively at HIV infected patients attending the genito-urinary medicine clinic to ascertain the incidence of oesophageal symptoms, the diagnostic value of various investigations and the results of treatment.

\section{Methods}

\section{PATIENTS}

At the Genito-Ürinary Medicine clinic of St Stephen's Hospital we currently follow more than $1500 \mathrm{HIV}$ infected patients, of whom 294 developed AIDS between January 1982 and December 1987. Ninety per cent of these patients are self-referrals, and only $45 \%$ of the patients have a general practitioner. In the 18 months to December 1987, 154 consecutive patients attending this clinic who were HIV antibody positive and had gastrointestinal symptoms, were carefully questioned about dysphagia and odynophagia by two of us (GMC and BGG). The patients were subsequently grouped according to the current classification of HIV infected patients of the Centres for Disease Control."

All patients with oesophageal symptoms were endoscoped and material for cytological examination was obtained using a standard endoscopic brush. The cytological samples were examined independently by an experienced technician and a senior member of the medical staff. Two direct smears were prepared, one being spray fixed using 'smear fix' (VALE), and the other being air dried. The slides were then

Table 1 AIDS diagnoses in 48 patients with oesophageal symptoms

\begin{tabular}{|c|c|c|}
\hline AIDS diagnosis & $\begin{array}{l}\text { Diagnosis of } \\
\text { AIDS before } \\
\text { endoscopy }\end{array}$ & $\begin{array}{l}\text { Diagnosis of } \\
\text { AIDS by } \\
\text { endoscopy }\end{array}$ \\
\hline \multicolumn{3}{|l|}{ Opportunistic infections } \\
\hline Candida & - & 16 \\
\hline $\begin{array}{l}\text { Mycobacterium avium intracellulare } \\
\text { (and Kaposi's sarcoma) }\end{array}$ & 1 & - \\
\hline Pneumocystis carinii pneumonia & 7 & - \\
\hline Cryptosporidiosis & 3 & - \\
\hline Herpes simplex virus & - & 1 \\
\hline Cytomegalovirus & - & 1 \\
\hline $\begin{array}{l}\text { Tumour } \\
\text { Kaposi's sarcoma ( } \pm \text { Pneumocystis } \\
\text { carinii pneumonia) }\end{array}$ & 18 & - \\
\hline \multicolumn{3}{|l|}{ Other } \\
\hline leukoencephalopathy & 1 & - \\
\hline
\end{tabular}

sterilised by immersion in $2 \%$ glutaraldehyde (AESP) solution for 20 minutes. The spray fixed slide was then stained using Papanicolaou's method and the air dried slide stained with May-Grünwald Giemsa. The stained slides were then screened for fungal elements and both herpetic and CMV inclusions. The endoscope brush was rinsed in saline and this fluid was examined under light microscopy for fungal elements.

Biopsies of oesophageal mucosa were taken from several sites via the endoscope and at least four extra samples were taken from around the edge of any ulcer present. The biopsies were fixed in $15 \%$ formal saline, embedded in paraffin by vacuum impregnation and $4 \mu \mathrm{m}$ sections were cut from at least three levels and routinely stained with haematoxylin and eosin, periodic acid-Schiff for fungi and ZiehlNeelsen stains for mycobacteria.

Thirty four of the 48 patients had a double contrast barium swallow and barium meal within 24 hours of endoscopy.

DIAGNंOSIS

The diagnosis of cytomegalovirus or herpes oesophagitis was confirmed by characteristic inclusion bodies seen within the nucleus or cytoplasm. Candidiasis was confirmed either on cytology or histology.

\section{Results}

Forty eight of the 154 patients examined had oesophageal symptoms: two were women aged 24 and 29 , who acquired HIV disease heterosexually. The rest were male homosexuals (age range 19-54 years, average 28). AIDS (Group IV $\mathrm{C}_{2}$ ) was diagnosed as a result of endoscopy in 18 patients and nine of these developed another AIDS diagnosis after endoscopy (Table 1).

\section{CAUSES OF OESOPHAGEAL DISEASE}

The most common cause of oesophageal disease was candidiasis, which was the only identified cause of symptoms in 26 patients and was found with other pathology in 12 (Table 2). Discrete ulceration of the oesophagus was identified in 10 patients at initial presentation, although candida was also present in seven. In three of these 10 patients the ulceration was shown histologically to be caused by cytomegalovirus and in four herpes simplex virus. Two other patients who had widespread aphthous ulceration of the mouth had similar ulcers in the gullet which showed the histological features of aphthous ulceration. The final patient had a peptic ulcer of the oesophagus with surrounding inflammation and typical histological changes. One patient sequentially developed herpetic ulceration associated with candidiasis, 
Table 2 Causes of oesophageal disease in AIDS in 48 patients

Single pathology

Candidiasis

Kaposi's sarcoma (KS)

Aphthous ulceration

Complex pathology (Candidiasis + another lesion)

Kaposi's sarcoma

Cytomegalovirus (CMV)

HSV

Aphthous ulceration

Peptic ulceration

HSV +CMV + aphthous ulceration

Mycobacterium avium intracellulare

Unknown pathology
Herpes simplex (HSV)

Table 3 Results of double,contrast barium swallow examination in 34 patients with AIDS and oesophageal symptoms

\begin{tabular}{|c|c|c|}
\hline Cause of oesophageal disease & $\begin{array}{l}\text { Pätients } \\
\text { studied }(n)\end{array}$ & $\begin{array}{l}\text { Reported as } \\
\text { abnormal (n) }\end{array}$ \\
\hline \multicolumn{3}{|l|}{ Opportunistic infections } \\
\hline Candida & 17 & 8 \\
\hline Mycobacterium avium intracellulare & $1^{*}$ & 1 \\
\hline Cytomegalovirus & $3^{* \dagger}$ & 1 \\
\hline Herpes simplex virus $\}$ discrete ulcer & $2^{*}$ & $i$ \\
\hline \multicolumn{3}{|l|}{ Tumour } \\
\hline Kaposi’s sarcoma & $5+$ & 1 \\
\hline \multicolumn{3}{|l|}{ Other } \\
\hline Aphthous\}discrete ulcer & 1 & 0 \\
\hline Peptic \}discrete ulcer & $1^{*}$ & 1 \\
\hline Unknown & 4 & 0 \\
\hline
\end{tabular}

*Indicates a patient with candidiasis in addition.

followed by cytomegalovirus ulceration and then aphthous ulceration.

In one patient with candidiasis and extensive oesophagitis and gastritis, acid fast bacilli were shown in the mucosa histologically and $\mathrm{Myco}$ bacterium avium intracellulare was subsequently cultured. In seven patients, typical lesions of Kaposi's sarcoma were seen, four having candidiasis in addition. No cause for the oesophageal symptoms was identified in four patients despite endoscopy, barium swallow, cytopathology, and histopathology.

\section{ENDOSCOPIC APPEARANCE}

The appearance of candidiasis of the oesophagus was simple to recognise and in 25 of the 38 cases a white membrane extended over the whole mucosa. In the other 13 patients, islands of candidiasis were seen with surrounding inflammation. The appearance of the four patients with herpes oesophagitis was similar to typical perioral herpes with superficial fluid filled vesicles. The aphthous ulcers in three patients were superficial spreading lesions with an irregular edge. The four cytomegalovirus ulcers were all deep linear ulcers up to $5 \mathrm{~cm}$ in length. Two had raised edges like an oesophageal carcinoma.

\section{CYTOLOGY AND HISTOLOGY}

Candidiasis was confirmed in all cases either by cytology or histology, but in only eight cases were both investigations positive. In 18 patients cytology only was positive and in a further 12 there was only histological evidence of infection. The fungus was found in saline rinses of the cytology brush in all eight cases where this test was additionally performed. In five of the 10 patients with oesophageal ulcers, a firm histological diagnosis was made from the initial endoscopy. The procedure was repeated in those patients in whom no diagnosis was found, and in two of these, histological evidence of cytomegalovirus was seen and the other three patients were assumed to have aphthous ulceration.

\section{RADIOLOGY}

In 34 of these 48 patients a barium swallow preceded any treatment and followed endoscopy within 24 hours (Table 3). More than half of those with oesophageal candidiasis only showed no radiological abnormality. Four of five patients with oesophageal $\mathrm{KS}$ were reported as normal (all four had flat plaque lesions of Kaposi's sarcoma). Of the seven patients with oesophageal ulcers who had a barium swallow, four were normal. Three of eight patients with candidiasis and another lesion had abnormal radiography but dual pathology was not suspected.

SYMPTOMS AND SIGNS

The symptoms of oesophageal disease were unusual when compared with common gastrointestinal causes. Oesophageal pain for both liquids and solids was always present and dysphagia occurred in 32 of the 48 patients. Two patients had severe non-specific chest pain, not related to swallowing, which resolved when the oesophageal disorder was treated. The oesophageal symptoms produced a disinclination to eat resulting in severe weight loss in only two patients.

Twenty seven of 38 patients with oesophageal candidiasis had buccal involvement including all 26 with candidiasis as the sole cause of symptoms. The four patients with herpetic oesophagitis also had perioral ulcers and all seven patients with Kaposi's sarcoma had disease involving the palate as well as extensive skin involvement. Three of the four patients with cytomegalovirus oesophagitis also had evidence of cytomegalovirus infection elsewhere (retina and colon (one), colon (two)). 


\section{Candidiasis}

In all 26 patients with oesophageal candidiasis only, symptoms responded to Ketoconazole $200 \mathrm{mg}$ bd within three days. Four were re-endoscoped three days after starting ketoconazole and none showed visible or subsequent pathological evidence of candida infection. In five patients, ketaconazole was stopped after 10 days, and in all, symptoms reappeared within a month but responded to its reintroduction. Liver function tests have been carefully monitored in all these patients for an average of six months (range 1-13) but no unexplained abnormality has been found.

\section{Cytomegalovirus infection}

Three of the four oesophageal ulcers caused by cytomegalovirus were treated with foscarnet (phosphonoformate, Astra Pharmaceuticals compassionate use only) in a dose of $20 \mathrm{mg} / \mathrm{kg}$ iv bolus over 20 minutes followed by a continuous infusion of $28.3-273.6 \mathrm{mg} / \mathrm{kg}$ over 24 hours dependent on renal function, for a period of two weeks. Two of these three patients have remained well for six months, and the third patient died six months after diagnosis, without oesophageal symptoms. The fourth patient died of a bowel perforation caused by cytomegalovirus colitis, before treatment could be started.

\section{Herpes simplex virus infection}

The four patients with herpetic oesophagitis were treated with intravenous acyclovir $(10 \mathrm{mg} / \mathrm{kg})$ intravenously for 10 days and the oesophageal mucosa healed completely in three.

\section{Kaposi's sarcoma}

Four of the seven patients with Kaposi's sarcoma of the oesophagus were treated with single agent etoposide (VP 16) (200 mg orally, daily for three days in three weekly cycles), but this failed to improve the oesophageal symptoms and they died within a maximum of 10 months of disseminated discase.

\section{Treatment with $\mathrm{H}_{2}$ antagonists}

Nine of the 10 patients with ulceration of the oesophagus were initially treated with ranitidine 300 $\mathrm{mg}$ tds for a minimum period of a week before other therapy, but in only one case (with histological changes of peptic ulcer disease) was there improvement in the symptoms.

\section{Complex oesophageal disease}

One patient with multiple oesophageal pathology

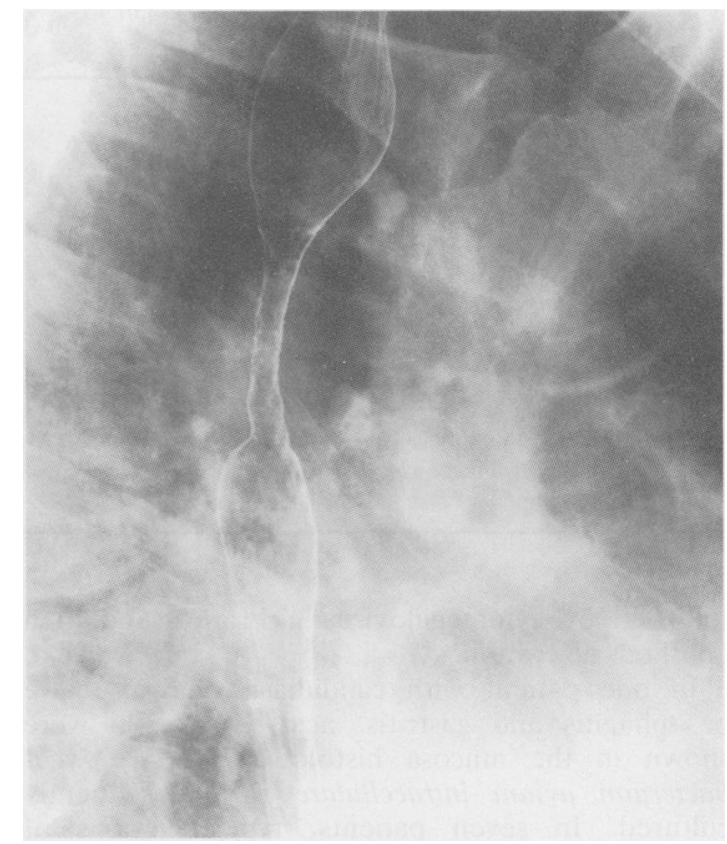

Fig. 1 Oesophageal stricture in a patient with herpes simplex virus and candidiasis of the oesophagus.

initially presented with buccal candidiasis and dysphagia, and was treated with ketoconazole. The dysphagia failed to resolve, however, and she developed perioral herpes and herpetic lesions of the oesophagus. These were treated with acyclovir intravenously without response, and subsequent endoscopy revealed macroscopically different ulcers which were shown histologically to be caused by cytomegalovirus infection. These ulcers were treated with foscarnet intravenously but her dysphagia remained a serious problem such that she had to be fed via a nasogastric tube. She then developed aphthous ulceration of the mouth and a further endoscopy revealed aphthous like ulcers in the gullet. She was treated with thalidomide ( $100 \mathrm{mg}$ daily) and over three days responded well. The pain from swallowing her saliva, previously requiring large doses of morphine, resolved. She was able to swallow normally and remained well for seven months, before succumbing to CMV colitis.

A further patient had evidence of candida and herpes infection of the oesophagus at endoscopy and was treated with both ketoconazole and acyclovir. Although he made a good clinical recovery, repeat endoscopy still revealed extensive inflammation. The patient discontinued maintenance treatment and three months later re-presented with severe dysphagia, and a stricture was shown on barium swallow 

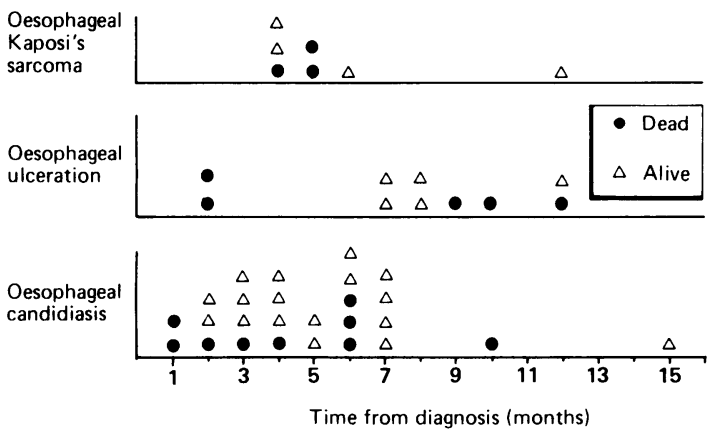

Fig. 2 Overall survival of patients with candidiasis, various ulcerating lesions and Kaposi's sarcoma of the oesophagus in AIDS. (Only 43 patients are shown, because those with unknown pathology (four) and one patient with MAI infection and candidiasis have not been included.)

(Fig. 1). This was successfully dilated and he is well three months later.

OVERALL SURVIVAL

None of these patients died of oesophageal disease but the average survival from its onset was only five months in the 44 patients with defined pathology (Fig. 2).

\section{Discussion}

Retrospective studies have analysed the causes of oesophageal symptoms in immunocompromised patients, " but we are unaware of any other studies in which their frequency has been prospectively evaluated. A formal protocol was not used to assess symptoms but all the patients were interviewed by two trained gastroenterologists as part of a larger study of the gastroenterological manifestations of HIV disease. The cause of oesophageal symptoms was similar to those found in other series of AIDS patients, " although cryptosporidial oesophagitis was not seen. ${ }^{2}$ One study has shown that buccal and oesophageal ulceration may occur at the time of acquisition of HIV infection. It is of interest that retrovirus like particles were seen budding from the gut mucosa at the edge of these ulcers but the appearances have not been reported in advanced disease..$^{13}$

The oesophageal symptoms in this study were dissimilar from that seen in general gastroenterology with pain and difficulty with swallowing for both solids and liquids.

Although the symptom complex did not help to determine the cause of oesophageal pathology in our patients inspection of the mouth was often helpful. Thus nearly all the patients with candidiasis and all those with herpes infection or Kaposi's sarcoma had involvement either of the buccal mucosa or palate.

The cause of oesophageal symptoms was usually obvious at endoscopy. The appearance of candidiasis is typical and is now a sufficient criteria for the diagnosis of AIDS by the Centres for Disease Control without the need for pathological confirmation. ${ }^{10}$ The various causes of ulcerating lesions are easy to identify at endoscopy as they have different macroscopic characteristics. It is often difficult to determine whether Kaposi's sarcoma of the gullet is responsible for symptoms as it was frequently associated with candidiasis, however, in three cases it was the sole finding.

Histological confirmation of the endoscopic diagnosis may be quite difficult. Candidiasis can be confirmed histologically but the hyphae may be washed from the mucosal surface during processing. Cytology may also occasionally fail to confirm the presence of the fungus. Perhaps the easiest approach is by microscopy of a saline rinse of cytology brushes. The confirmation of the diagnosis of cytomegalovirus infection which depends upon inclusion bodies in histological specimens is particularly difficult in the oesophagus, where it is only possible to take small biopsies. In two of our patients the diagnosis was found only at a second endoscopy.

Treatment of oesophageal disease in HIV infected individuals is worthwhile as a symptomatic response can nearly always be obtained. Ketoconazole should be continued long term once the diagnosis of oesophageal candidiasis is made because in our experience, relapse occurred when therapy was stopped. In a previous study which has indicated possible resistance to ketoconazole therapy, other potential causes of symptoms were not rigorously excluded. ${ }^{1+}$ As other data ${ }^{15}{ }^{16}$ have shown intravenous treatment with acyclovir is required for severe genital herpes infection, this route of administration was also used for oesophageal herpes. Subsequently it has been our practice to continue acyclovir orally as prophylaxis.

Foscarnet appears to be an effective treatment of cytomegalovirus oesophagitis and has produced prolonged remissions in all three patients in this study. This is surprising, because other workers' experience with the use of this drug in cytomegalovirus retinitis has been that relapse rapidly follows discontinuation of therapy. ${ }^{17}$ It has been previously reported that this drug is not effective in the treatment of cytomegalovirus colitis and one case of oesophageal ulceration did not respond to therapy. ${ }^{18}$ An alternative treatment, gangciclovir, produced resolution of symptoms in four of five patients, with presumed cytomegalovirus oesophagitis, although in these cases biopsy confirmation of the diagnosis was not obtained. ${ }^{19}$ 
Thalidomide is an effective drug for the treatment of aphthous ulceration of the mouth secondary to Behçet's syndrome,"l" and it produced a dramatic improvement in one of our patients with oesophageal pain and oesophageal ulceration.

The aim of treatment of Kaposi's sarcoma in AIDS is a palliation of symptoms rather than prolongation of life. The prognosis of patients with visceral Kaposi's sarcoma is particularly poor and in our own experience treatment with etoposide has produced no improvement in oesophageal symptoms.

As radiology was not always helpful in diagnosing the cause of oesophageal symptoms, endoscopy is the best diagnostic procedure in HIV infected patients. Endoscopy may be associated with a small risk to health care staff. There are, however, no recorded cases of endoscopic staff seroconverting as a result of occupational exposure. The seroconversion rate resulting from needlestick injury is only $0.5 \%$."

The present DHSS guidelines dealing with endoscopy in groups of patients who are at risk of an HIV infection are cumbersome, including an hour's presoaking with glutaraldehyde before washing, followed by a three hour immersion as a sterilisation procedure. These guidelines are now being reviewed with a much greater emphasis on immediate, thorough washing, and a much shorter soaking in glutaraldehyde, which should enable endoscopy procedures in high risk patients to be undertaken more easily. ${ }^{21}$

It is possible to treat many HIV antibody positive patients with oesophageal symptoms empirically, without a definitive diagnosis. Thus patients with buccal candidiasis and painful swallowing can be given ketoconazole, reserving endoscopy for those who fail to respond. While this approach may be necessary in centres with large numbers of patients and limited facilities, it would be a major departure from normal clinical practice. We recommend endoscopy for all patients with oesophageal symptoms as one third will have an AIDS diagnosis confirmed for the first time and one third will have more than one pathological cause. Half the patients with AIDS, diagnosed with oesophageal symptoms, will be dead within five months.

We would like to thank the patients and staff of Thomas Macauley Ward and the John Hunter Clinic, St Stephen's Hospital, and Linda Stannard and Christina Derry for their help in typing this paper.

\section{References}

1 Kaposi`s sarcoma and Pncumocystis pneumonia among homosexual men - New York City and California. M.MWR 1981: 30: 305-8.

2 Acquired Immunodeficiency Syndrome: United Kingdom: 1982-December 1987. CDR 88/(01, 8th January 1988.

3 Gottleib MS. Groopman JE, Weinstein WM. Fahey JL. Detels R. The Acquired Immunodeficiency Syndrome. Ann Intern Med 1983; 99: 208-20.

4 Green JB. Sidhu GS. Lewin S, et al. Mycobacterium Avium Intracellulare: a cause of disseminated lifethreatening infection in homosexuals and drug abusers. Ann Intern Med 1982: 97: 539-46.

5 Fauci AS. Macher AM. Longo DL, et al. Acquired Immunodeficiency Syndrome, epidemiologic, clinical immunologic and therapeutic considerations. Ann Intern Med 1984: 100: 92-106.

6 Friedman-Kien AE, Laubenstein LJ. Rubinstein P. et al. Disseminated Kaposi's sarcoma in homosexual men. Ann Intern Med 1982: 96: 693-7(0).

7 Emery CD, Wall SD, Federle MP. Sooy CD. Pharyngeal Kaposi's sarcoma in patients with AIDS. AJR 1986; 147: 919-22.

8 Turchi R. Bacciu S, Jemmi G, Tuberti E. Anatomoclinical considerations on a case of Kaposi's sarcoma. Acta Biomed Ateneo Parmense 1986; 57: 63-9.

9 Revision of the CDC surveillance case definition for Acquired Immunodeficiency Syndrome. MMWR 1987: 36: $1 \mathrm{~S}$.

10 CDC classification system for human T-lymphotropic virus type $I I / /$ lymphadenopathy-associated virus infections. Ann Intern Med 1986; 105: 234-7.

11 Wheeler RR, Peacock JE, Gruz JM, et al. Ocsophagitis in the immunocompromised host: role of endoscopy in diagnosis. Rev Infect Dis 1987: 9: 88-95.

12 Kazlow PG. Shah K. Benkov KJ, Dische R, Leleiko NS. Oesophageal cryptosporidiosis in a child with Acquired Immunodeficiency Syndrome. Gastroenterology 1986; 91: 1301-3.

13 Rabeneck L, Boyko WJ, McClean DM, McLcod WA. Wong KK. Unusual oesophageal ulcers containing enveloped virus-like particles in homosexual men. Gastroenterology 1986; 90: 1882-9.

14 Tavitian A, Raufman JP, Rosenthal LE, Weber J, Webber CA. Dincsoy HP. Ketoconazole-resistant candida oesophagitis in patients with Acquired Immunodeficiency Syndrome. Gastroenterology 1986; 90: $443-5$.

15 Corey L, Fife KH, Benedetti JK, et al. Intravenous Acyclovir for the treatment of primary genital herpes. Ann Intern Med 1983; 98: 914-21.

16 Mindel A, Adler NW, Sutherland S, Fiddian AP. Intravenous Acyclovir treatment for primary genital herpes. Lancet 1982; i: 697-700.

17 Walmsley SL, Chew E, Fanning MM, et al. Treatment of cytomegalovirus retinitis with Trisodium Phosphonoformate (Foscarnet). Paper presented at III International Congress on AIDS, Washington [Abstracts], 1987.

18 Weber JN. Thom S, Barrison I, et al. Cytomegalovirus colitis and oesophageal ulceration in the context of AIDS: clinical manifestations and preliminary report of treatment with Foscarnet. Gut 1987; 28: 482-7.

19 Chachoua A. Dieterich D, Krasinski K, et al. 9-(1,3- 
dihydroxy-2-propoxymethyl guanine) Ganciclovir in the treatment of cytomegalovirus gastrointestinal disease with the Acquired Immunodeficiency Syndrome. Ann Intern Med 1987; 107: 133-7.

20 McCray E. The Co-operative Needlestick Surveillance Group. Occupational risk of the Acquired Immuno- deficiency Syndrome among health care workers. N Engl J Med 1986; 314: 1127-32.

21 Working Party of British Socicty of Gastroenterology. Cleaning and disinfectant of equipment for gastrointestinal flexible endoscopy: interim recommendations. Gut 1988: 29: 11.34-51. 